\title{
Mars Observer's Global Mapping Mission
}

\section{A. L. Albee and D. F. Palluconi \\ California Institute of Technology, Pasadena, California PAGES 1099, 1107}

The Mars Observer mission, scheduled for launch in September 1992, will provide an orbital platform at Mars from which the entire Martian surface and atmosphere will be observed beginning in late 1993. Mars Observer will extend the exploration and charac terization of Mars by providing new and systematic measurements of the surface and atmosphere of the planet. These measurement will be made from a low-altitude polar orbiter over a period of one Martian year (687 Earth days), permitting repetitive observations of the surface and of the seasonal variations of the atmosphere. The mission is being designed in a manner that will provide new and valuable scientific data at a significant reduction in cost and operational complexity.

The scientific objectives for the mission emphasize qualitative and quantitative determination of the elemental and mineralogical composition of the surface; measurement of the global surface topography, gravity field, and magnetic field; and the development of synoptic data base of climatological conditions. This mission will provide a basic global understanding of Mars as it exists today and will provide a framework for understanding its past.

The formal scientific objectives of this geoscience and climatological mission are to determine the global elemental and mineralogical character of the surface material; define globally the topography and gravitational field; establish the nature of the magnetic field; determine the time and space distribution, abundance, sources, and sinks of volatile material and dust over a seasonal cycle; and explore the structure and aspects of the circulation of the atmosphere.

These first-order scientific objectives can be addressed within the framework of a low-cost orbital mission. All five objectives involve global mapping. For the geoscience objectives, this mapping is mainly time-independent and therefore two-dimensional: latitude and longitude. For many climatology objectives, the mapping is four-dimensional: latitude, longitude, altitude and season. As a result of this mission we should have a system atic global characterization of Mars today. This characterization will help us to understand the geologic and climatologic history of Mars, the evolution of its interior and sur

Cover. By the end of 1993, the Mars Observer spacecraft will begin mapping the surface and atmosphere of Mars, extending the exploration and characterization of the planet by providing new and systematic measurements over a period of one Martian year. See "Mars Observers' Global Mapping Mission" by A. L. Albee and D. F. Palluconi, this issue.

MISSION

PHASE: RECORD: face, and provide a basis for comparison with Venus and Earth.

\section{Mission Description}

Mars Observer will be launched by a Titan III, built by Martin Marietta, with an upper Transfer Orbit Stage from Orbital Sciences Corporation. After the 11-month transit the spacecraft will be injected into an elliptical or bit around Mars with periapsis near the north pole. The orbit is then adjusted through a se ries of maneuvers to a near-circular, sun-synchronous (2:00 A.M./P.M.), low altitude, near-polar orbit. Due to the non-uniformity of the gravity field, the altitude varies from $376 \mathrm{~km}$ near the south pole to $430 \mathrm{~km}$ near the north pole. During the Martian year in this mapping orbit the instruments acquire data in a systematic program of global mapping. At the end of the mission the spacecraf can be boosted to a permanent quarantine or bit.

The mapping timeline, relative to the Martian seasons and the likely dust storm period is summarized in Figure 1. The Mars orbit insertion (MOI) period ends just before solar conjunction and the beginning of the dust storm period. Since it is scientifically important to make observations for an entire global mapping cycle ( 26 days) before the onset of a major dust storm, it is hoped that the fuel margin will permit a shorter MOI phase than shown

The playback data rate for a $\mathbf{1 0}$-hour link varies by a factor of 4 with Earth-Mars distance during the mission. The continuous re- corded data rate to the tape recorders is about one-fifth the playback rate for a 10 hour link to a $34 \mathrm{~m}$ NASA Deep Space Network tracking station. The normal sequence of collecting scientific data will be to record continuously for 24 hours and then to play the data back in one 10-hour link. The mapping orbit is a 117-minute orbit with a 7 -day repeat cycle. As a result, the planet is repeat edly mapped in 26-day cycles with a $58.6 \mathrm{~km}$ nominal path separation. Orbit trim adjustments make it possible to obtain uniform coverage during the course of the mission with an ultimate spacing of ground tracks at the equator or $3.1 \mathrm{~km}$

The Mars Observer spacecraft provides a three-axis stabilized, nadir-oriented platform for continuous observations of Mars by the science instruments (Figure 2). The spacecraft is being built by the General Electric Astro-Space Division. The Gamma Ray Spec trometer and Magnetometer sensor assemblies are mounted on individual booms on the spacecraft. All other instruments are rigidly mounted to the spacecraft structure. No movable scan platform is provided; the spacecraft is continuously nadir pointed, rotating at the orbital rate. Those instruments that re quire scanning or multiple fields of view have internal scanning mechanisms. The footprints for the instruments are shown in Figure 3.

\section{Experiments and Instruments}

The instruments for Mars Observer closely match the scientific objectives. Collectively the instruments cover much of the electromagnetic spectrum and form a highly complementary set. Each instrument produces welldefined sets of measurements which address specific major objectives, but nearly every data set also contributes to a much wider variety of scientific investigations. Five interdisciplinary scientists have been selected in addition to the instrument teams in order to exploit the strong synergism in the data sets. Moreover, participating scientists, including 10 from the Soviet Union, will be added after launch to further exploit the data returned
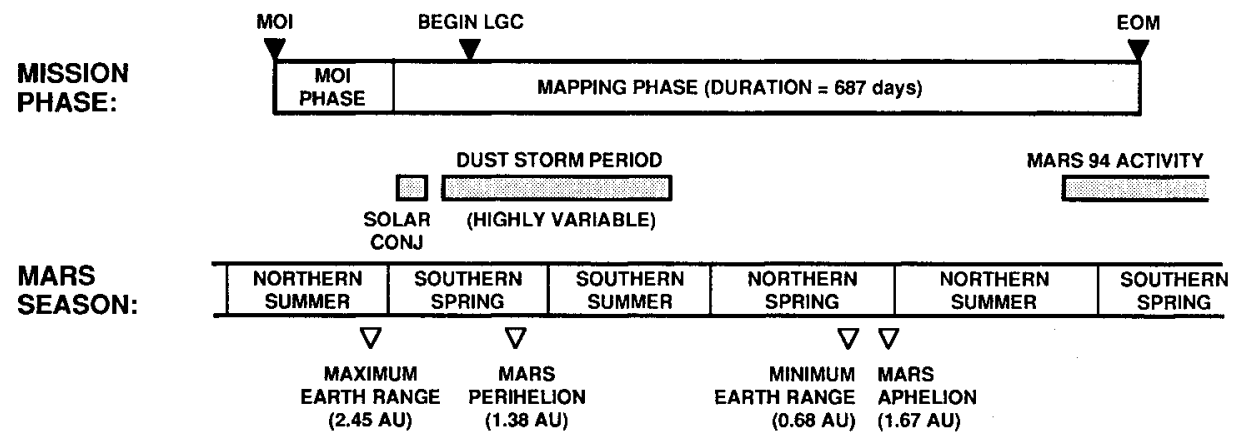

DATA RATES

PLAYBACK:

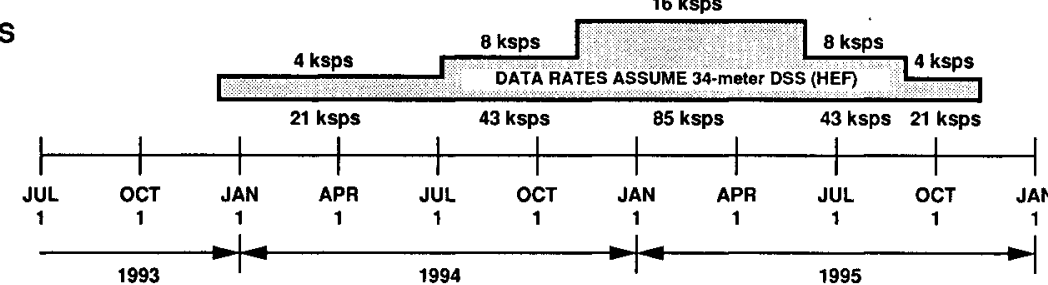

Fig, 1. Timelines for the mapping phase of the Mars Observer mission. 


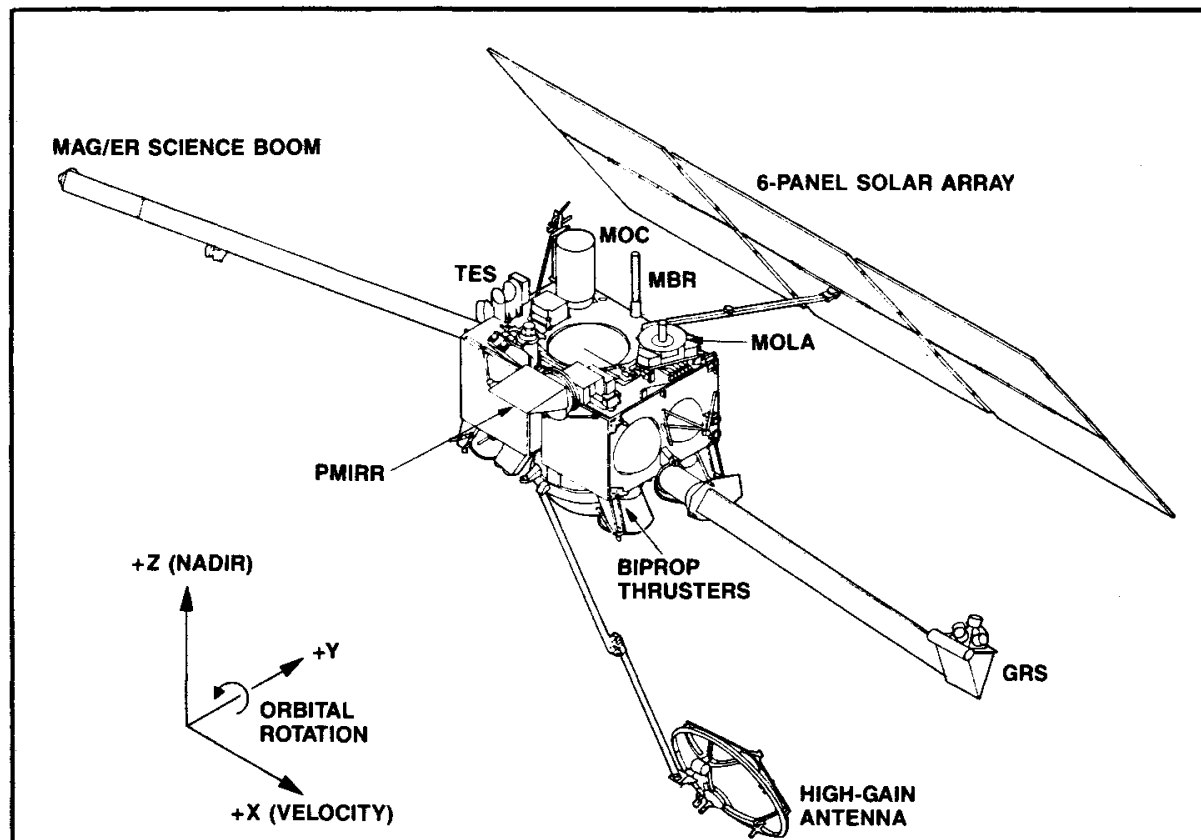

Fig. 2. The Mars Observer spacecraft and its payload in the fully deployed configuration of its mapping orbit at Mars.

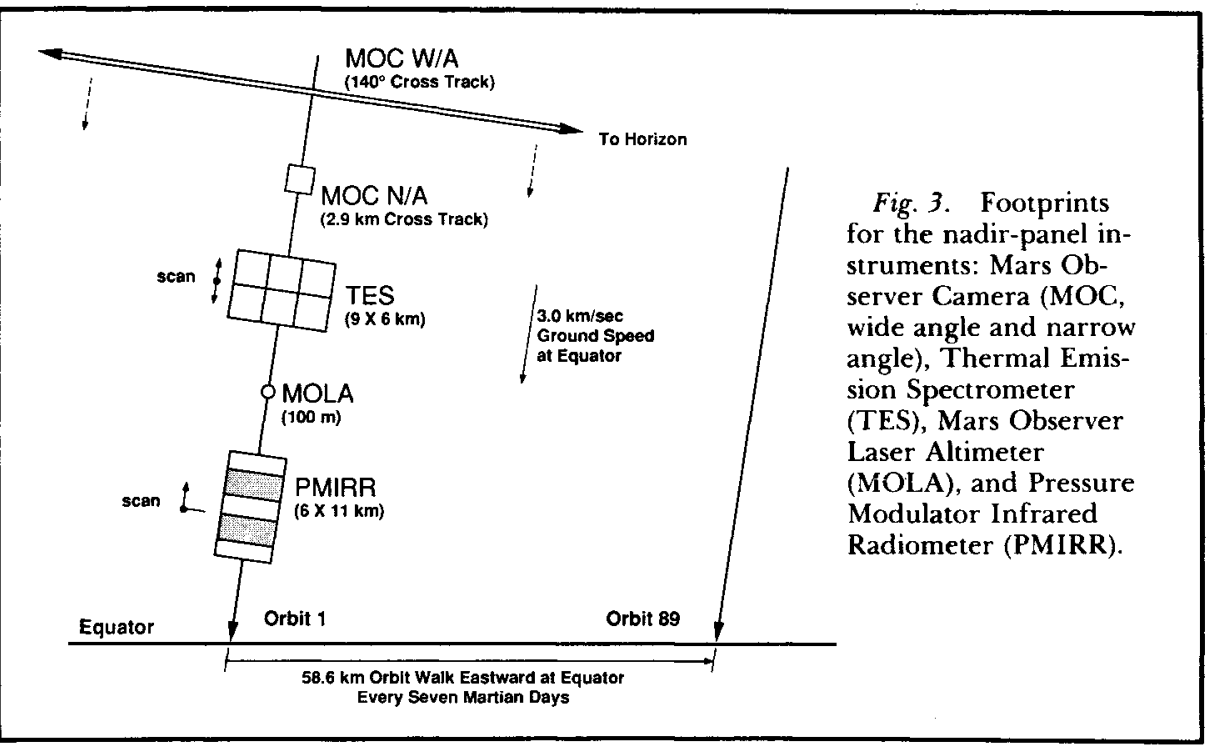

from the mission. Each of the instruments and their experimental objectives are described below.

Gamma Ray Spectrometer and Neutron Detector (GRS). The team leader for the GRS experiment is W. Boynton of the University of Arizona. This boom-mounted instrument has a high spectral-resolution germanium detector cooled to $100 \mathrm{~K}$ by a passive radiator. It will measure the intensities of characteristic gamma-ray lines that emerge from the Martian surface within the energy range 0.2 to 10 $\mathrm{MeV}$. A boron-doped plastic scintillator will serve as a charge-particle shield and as a neutron detector to determine the abundance of hydrogen.

Key objectives of this investigation are to determine the elemental composition of the surface of Mars with a spatial resolution as high as a few hundred kilometers through measurements of gamma-rays excited at the
Martian surface and albedo neutrons $(\mathrm{H}, \mathrm{O}$, $\mathrm{Mg}, \mathrm{Al}, \mathrm{Si}, \mathrm{S}, \mathrm{C}, \mathrm{K}, \mathrm{Ca}, \mathrm{Fe}, \mathrm{Th}, \mathrm{U})$ and to determine the arrival times and spectra of gamma-ray bursts.

Mars Observer Camera (MOC). The principal investigator for MOC is $\mathrm{M}$. Malin of Arizona State University. The instrument is a line scan camera, which incorporates both wideangle and narrow-angle optics for producing global coverage $(7.5 \mathrm{~km} / \mathrm{pixel})$, selective moderate-resolution images $(480 \mathrm{~m} / \mathrm{pixel})$, and very selective high-resolution $(1.4 \mathrm{~m} / \mathrm{pixel})$ images. global synoptic views of the martian atmosphere (clouds) and surface to study meteorological, climatological, and related surface changes on a daily basis; monitor surface and atmosphere features at moderate resolution for changes on time scales of hours, days, weeks, months and years; and systematically
Objectives of this investigation are to obtain examine local areas at extremely high spatial resolution in order to quantify surface/atmosphere interactions and geological processes.

Thermal Emission Spectrometer (TES). The principal investigator for TES is P. Christensen of Arizona State University. The instrument is a Michelson interferometer that covers the spectral range 6.25 to 50 micrometers with 5-10 wavenumber spectral resolution. Separate solar reflectance $(0.3$ to 3.9 micrometers) and broad band radiance (0.3 to 100 micrometers) channels are included for radiation balance measurements. It has six 8.3 mrad fields of view, each with $3 \mathrm{~km}$ spatial resolution at nadir.

Objectives of this investigation are to determine and map the composition of surface minerals, rocks and ices; study the composition, particle size, and spatial and temporal distribution of atmospheric dust and of condensate clouds; study the growth, retreat and total energy balance of the polar cap deposits; measure the thermophysical properties of the Martian surface (thermal inertia, albedo) that can be used to derive surface particle size and rock abundance; and determine atmospheric temperature, pressure, water vapor, and ozone profiles, and seasonal pressure variations.

Pressure Modulator Infrared Radiometer (PMIRR). The principal investigator for PMIRR is D. McCleese of the Jet Propulsion Laboratory. The instrument is a limb, off-nadir and nadir scanning radiometer. Measurements are made in 9 spectral bands with 5 filter channels and 2 pressure modulator cells (one containing carbon dioxide, the other water vapor). The detectors are cooled to $80 \mathrm{~K}$ by a passive radiator.

The objectives of this investigation are to map the three-dimensional and time-varying thermal structure of the atmosphere from the surface to $80 \mathrm{~km}$ altitude; map the global, vertical and temporal variation of atmospheric dust and condensates; map the seasonal and spatial variation of atmospheric pressure and the vertical distribution of atmospheric water vapor; and monitor the polar radiation balance.

Mars Observer Laser Altimeter (MOLA). The principal investigator for MOLA is D. Smith of Goddard Space Flight Center. The instrument is a diode-pumped 1.06 micrometer $\mathrm{Nd}$ :YAG laser transmitter and a $50 \mathrm{~cm}$ diameter parabolic receiver, which provide $100 \mathrm{~m}$ diameter footprints with $2 \mathrm{~m}$ vertical precision at 10 pulses per second (about $300 \mathrm{~m}$ spacing).

The key objectives are to determine a global, geodetically referenced $0.2^{\circ} \times 0.2^{\circ}$ topographic grid to a vertical precision of $30 \mathrm{~m}$ to address global problems in geology, geophysics, and atmospheric circulation; and measure globally distributed topographic profiles with a vertical precision of $2 \mathrm{~m}$ to address local and regional geologic and geophysical problems.

Radio Science. The team leader for the Radio Science investigation is G. L. Tyler of Stanford University. The instrument is the spacecraft radio subsystem, $x$-band up and down, supplemented with an ultrastable oscillator to maximize the science during occultation exits. Radio occultation observations provide data on the atmosphere and radio Dopp- 
ler tracking of the spacecraft motion provides data on the gravitational field.

Key atmospheric and gravitational objectives are to determine profiles of refractive index, number density, temperature, and pressure at up to $200 \mathrm{~m}$ vertical resolution for the lowest few scale heights at high latitudes in both hemispheres on a daily basis fo a Martian year; characterize the small scale structure of the atmosphere and ionosphere develop a global, high-resolution model of the gravitational field; and determine both local and broad scale density-structure and stress state of the Martian crust and upper mantle.

Magnetometer and Electron Reflectometer. The principal investigator of the Magnetometer is M. Acuna of the Goddard Space Flight Center. The instrument has two triaxial fluxgate magnetometers and an electron reflectomete mounted on a 6-meter spacecraft boom. The magnetometer measures the components of the magnetic field, providing the orientation and magnitude of the ambient field. The electron reflectometer measures the energy spectrum and angular distribution of inciden and reflected electrons.

Key objectives of this investigation are to establish the nature of the magnetic field of Mars, map the Martian crustal remanent field using the fluxgate sensors and extend these in-situ measurements with the remote capability of the electron reflectometer sensor, and characterize the solar wind/Mars plasma interaction.

Mars Balloon Relay (MBR). The MBR radio system is being provided by the French space agency (CNES) to relay scientific telemetry from balloons or landers that will be deployed at Mars by the Soviet Mars 1994 mission. Work is proceeding on the Mars 94 mis sion despite current political problems. The MBR system will utilize the buffer memory of the Mars Observer Camera in order to great- ly enhance the telemetry from balloon-suspended experiments, which are expected to include imaging, atmospheric, and other instruments. This relay function would occur in late 1995 near the end of the nominal Mars Observer mission.

The interdisciplinary scientists with geoscience objectives are R. Arvidson of Washington University, M. Carr of the U. S. Geological Survey, Menlo Park, and L. Soderblum of the U. S. Geological Survey, Flagstaff. The interdisciplinary scientists with atmospheric and climatologic objectives are $A$. Ingersoll of the California Institute of Technology, B. Jakosky of the University of Colorado, and J. Pollack of Ames Research Center.

\section{Operations and Data Analysis}

The Mars Observer mission operations at the Jet Propulsion Laboratory (JPL) will be supported by the NASA Deep Space Network and the JPL Space Flight Operations Center. The science investigation teams will be remotely located at the home institutions of the principal investigators, team leaders, and other key science personnel. Workstations and electronic communication links will connect the mission planning and data analysis activities of these scientists, engineers, and mission managers.

The Mars Observer project will utilize data standards for packet telemetry and telemetry channel coding and will use a standard formatted data unit for data transfer among ground systems. Mission data will be stored in a project data base. Analysis data will consist of a record of each instrument's packet telemetry data provided as an experiment data record; spacecraft position and pointing information data available as a supplementary experiment data record; and related data such as spacecraft status, commands, data availability, and ancillary data. Planning products available will be the up-to-date mis- sion sequence plan, schedules and commanding opportunities, and orbit/viewing forecasts. Investigators and analysts will access the data base to participate in the planning process and to provide analysis products, including spacecraft and instrument performance and status, and higher-order data products, such as intermediate and final science products.

All of the instruments are "mapping" instruments in some sense. Efficient comparison of such data requires global digital data bases that are each accessible to end users and that can be manipulated without the assistance of technological experts. A sinusoidal equal-area projection will be used as a base. In this projection each parallel of latitude is an image line with its length scaled by the cosine of its latitude. A global digital image mosaic is being constructed from selected Viking images. Each pixel represents $1 / 256^{\circ}$ (about $230 \mathrm{~m}$ ); lower resolution versions can be readily produced by factors of negative pow ers of 2. Such a base and system is designed to allow efficient computer storage and management, user access, coregistration of data bases, rapid manipulation of data bases for effective analysis and interpretation, and inexpensive preparation of image maps on any desired projections. The data bases will be widely distributed, probably on digital (CDROM) disks

\section{Acknowledgments}

The research described here was carried out by the Mars Observer Project at the Jet Propulsion Laboratory, California Institute of Technology, under contract with the National Aeronautics and Space Administration. The authors are the project scientists for the Mars Observer mission and acknowledge the contribution of the entire project staff. The science objectives for the individual investigations are summarized from the science plans prepared by the individual investigators.

\section{Senate Recommends NSF Funding Increase, NASA Cuts \\ PAGE 1099}

The Senate VA, HUD, and Independen Agencies Appropriations Subcommittee chaired by Barbara Mikulski (D-Md.), recommended on September 12 to increase fiscal year 1991 funding of the National Science Foundation (NSF) by $\$ 27$ million above the level approved by the House. At the same time the subcommittee approved a spending level for the National Aeronautics and Space Administration (NASA) that is $\$ 855$ million below that recommended by the House

NSF funding under the Senate bill (H.R. 5158 ) would be $\$ 2.36$ billion, an increase of $14 \%$ over last year. Like the House bill, the Senate's bill eliminates funding for a gravitational wave observatory. Science education programs were increased to $\$ 322$ million, higher than both the House bill and President Bush's request.
The Senate bill reduces NASA's budget to $\$ 13.45$ billion, which is more than $\$ 1.5$ billion less than Bush requested. Recommended cuts include $\$ 863$ million from Space Station Freedom and $\$ 30$ million from the Moon/Mars initiative. A $\$ 50$ million reduction in funding for the Comet Rendezvous Asteroid Flyby (CRAF)/Cassini mission could mean the cancellation of the CRAF portion of the combined program. On the brighter side, funding for the Earth Probes satellite program was increased by $\$ 31$ million and basic Earth science research and modelling received an additional $\$ 17$ million.

The subcommittee's recommendations now await approval by the Senate, after which the bill goes to a House-Senate conference to resolve the differences between the two bills. If, however, a final bill is not signed by the President before the beginning of the 1991 fiscal year on October 1, automatic reductions imposed by the Gramm-Rudman-Hollings Act will take effect. For NSF, this would mean a possible $50 \%$ cut in funding to grantees. NASA could face cancellation of two or three scientific projects, such as CRAF and the Ad- vanced X-Ray Astrophysics Facility, and the possible suspension of space shuttle flights until 1993

\section{Saturn's 18th Moon \\ Located}

\section{PAGE 1099}

The discovery of a new moon orbiting Saturn brings to 18 the number of the planet's moons. Temporarily designated $1981 \mathrm{~S} 13$, the moon has a diameter of only $20 \mathrm{~km}$ and apparently creates the 320-km-wide "Encke's gap" in the planet's " $A$ " ring, its outermost major ring.

The small, bright object was found by Mark Showalter of the National Aeronautics and Space Administration, Ames Research Center, Moffett Field, Calif,, while analyzing images taken by the Voyager 2 spacecraft during its 1981 flyby of Saturn. Showalter used a computer program he wrote to sort through 30,000 images.

A "moonlet wake" pattern, resembling a motorboat wake, was first noticed in the ring 